\title{
ON A CLASS OF QUASICONFORMAL FUNCTIONS IN BANACH SPACES
}

\author{
SU-SHING CHEN
}

\begin{abstract}
A quasiconformal function $f$ on a domain $D$ in a complex Banach space $E$ is defined as a function on $D$ such that for every holomorphic mapping $\Phi$ from the unit disk $\Delta$ into $D$ the composite mapping $f \circ \Phi$ is quasiconformal in the usual sense. With respect to the Kobayashi-Kiernan pseudo distance on $D$, Schwarz's lemma, Liouville's theorem and the little Picard theorem are obtained for quasiconformal functions. A maximum modulus principle is also obtained for quasiconformal functions.
\end{abstract}

1. Introduction. In [4], P. J. Kiernan has proved Schwarz's lemma for $K$-quasiconformal mappings of Riemann surfaces. He has also obtained simple proofs of several well-known theorems for $K$-quasiconformal mappings, such as Liouville's theorem, Schottky's lemma and Picard's theorems. We extend some of his results to $K$-quasiconformal functions in complex Banach spaces. This class of functions contains the class of all holomorphic functions on a domain in a complex Banach space. (See [2] and [6].) In order to obtain Schwarz's lemma, we shall generalize S. Kobayashi's definition of pseudo distances on complex manifolds to complex Banach manifolds.

2. A class of quasiconformal functions. Among various definitions [1] of quasiconformal functions of a complex variable, we adopt the following definition. Let $\mu$ be a $C$-valued measurable function on a domain $D$ in $C$ with $|\mu| \leqq(K-1) /(K+1)$. Let $f$ be a continuous function with $L_{2}$ derivatives satisfying the Beltrami equation $f_{\bar{z}}=\mu f_{z}$ almost everywhere. Then $f$ is called a $K$-quasiconformal function on $D$. Here $f_{\bar{z}}=\frac{1}{2}(\partial f / \partial x-i \partial f / \partial y)$, $f_{z}=\frac{1}{2}(\partial f / \partial x+i \partial f / \partial y)$ and $K$ is a constant $\geqq 1$, called the dilatation of $f$.

Definition 1. A $K$-quasiconformal function $f$ on a domain $D$ in a complex Banach space $E$ is a function $f: D \rightarrow C$ on $D$ such that for each holomorphic mapping $\Phi: \Delta \rightarrow D$ from the unit disk $\Delta$ into $D$, the composite function $f \circ \Phi: \Delta \rightarrow C$ is $K$-quasiconformal in the above sense.

Received by the editors May 12, 1971 and, in revised form, May 27, 1972.

AMS (MOS) subject classifications (1970). Primary 32H15, 32H25.

Key words and phrases. Quasiconformal function, Kobayashi pseudo distance, Schwarz lemma, Liouville theorem, the little Picard theorem, maximum modulus principle.

(c) American Mathematical Society 1973 
It is obvious that holomorphic functions on $D([2]$ and [6]) are 1quasiconformal functions. We remark that results of [4] hold for quasiconformal functions $f \circ \Phi$ in Definition 1. $K$-quasiconformal functions of several complex variables are first defined by $S$. Hitotumatu in [3]. In addition to the condition in Definition 1, the function $f$ is assumed to be of class $C^{\mathbf{1}}$ in his definition. (See [3].)

3. Kobayashi-Kiernan pseudo distances. In this section, we define the Kobayashi-Kiernan pseudo distance on a complex Banach manifold. Let $\rho$ denote the Poincaré-Bergman distance on the unit disk $\Delta$, that is

$$
\tanh \frac{1}{2} \rho\left(z, z^{\prime}\right)=\left|z-z^{\prime}\right| /\left|1-\bar{z} z^{\prime}\right|,
$$

where $z, z^{\prime} \in \Delta$. Let $M$ be a complex Banach manifold. Given two points $p, q \in M$, we choose points $p=p_{0}, p_{1}, \cdots, p_{n-1}, p_{n}=q$ of $M$, points $a_{1}, \cdots, a_{n}, b_{1}, \cdots, b_{n}$ of $\Delta$ and holomorphic mappings $\varphi_{1}, \cdots, \varphi_{n}$ of $\Delta$ into $M$ such that $\varphi_{i}\left(a_{i}\right)=p_{i-1}$ and $\varphi_{i}\left(b_{i}\right)=p_{i}$, for $i=1, \cdots, n$. For each choice of points and mappings thus made, we consider the number $\rho\left(a_{1}, b_{1}\right)+\cdots+\rho\left(a_{n}, b_{n}\right)$. The Kobayashi pseudo distance $d_{M}(p, q)$ is defined as the infimum of $\rho\left(a_{1}, b_{1}\right)+\cdots+\rho\left(a_{n}, b_{n}\right)$ obtained in this manner for all possible choices.

Let $\tilde{\rho}$ be an arbitrary distance on $\Delta$. Replacing $\rho$ by $\tilde{\rho}$ in the above definition, we obtain a pseudo distance $\tilde{d}_{M}$, called the generalized Kobayashi pseudo distance on $M$. In [4], P. J. Kiernan has defined a distance $h_{\lrcorner, K}(K \geqq 1$ is a constant $)$ on $\Delta$ by

$$
\begin{aligned}
h_{\Delta \cdot K^{-}}\left(z, z^{\prime}\right) & =C_{K^{-}} \rho\left(z, z^{\prime}\right) & & \text { if } \rho\left(z, z^{\prime}\right) \geqq 1, \\
& =C_{K^{*}} \rho\left(z, z^{\prime}\right)^{1 / K} & & \text { if } \rho\left(z, z^{\prime}\right) \leqq 1,
\end{aligned}
$$

for each $z, z^{\prime} \in \Delta$ and $C_{K}$ a certain constant.

Definition 2. The Kobayashi-Kiernan pseudo distance $d_{M . K}$ on $M$ is the generalized Kobayashi pseudo distance with $\tilde{\rho}=h_{\lrcorner, K}$.

From the definition, it follows that $d_{M . K} \geqq d_{M I}$.

4. Schwarz's lemma and other theorems. In this section we shall obtain Schwarz`s lemma and some other theorems for $K$-quasiconformal functions on a domain in a complex Banach space $E$. We are indebted to P. J. Kiernan's paper [4] for the proofs of theorems here.

THEOREM 1 (SCHWARZ'S LEMMA). Let $f: D \rightarrow \Delta$ be a K-quasiconformal function from a domain $D$ in a complex Banach space $E$ into the unit disk $\Delta$. Then $f$ is distance decreasing with respect to the pseudo distance $d_{D, K}$ and the distance $d_{\lrcorner}$; that is

$$
d_{د}(f(p), f(q)) \leqq d_{D . K^{-}}(p, q) \text { for } p, q \in D .
$$


Proof. For $p, q \in D$, the pseudo distance $d_{D, K}(p, q)$ is the infimum of $h_{\Delta, K}\left(a_{1}, b_{1}\right)+\cdots+h_{\Delta, K}\left(a_{n}, b_{n}\right)$ over all possible choices of $a_{1}, \cdots, a_{n}$, $b_{1}, \cdots, b_{n} ; p_{0}=p, \cdots, p_{n}=q$ and holomorphic mappings $\varphi_{i}: \Delta \rightarrow D$ such that $\varphi_{i}\left(a_{i}\right)=p_{i-1}$ and $\varphi_{i}\left(b_{i}\right)=p_{i}$. Applying Schwarz's lemma (Theorem 2' of [4]) to $a_{i}, b_{i} \in \Delta$ and $f \circ \varphi_{i}: \Delta \rightarrow \Delta\left(f \circ \varphi_{i}\right.$ is $K$-quasiconformal), we have

Thus

$$
h_{\Delta, K}\left(a_{i}, b_{i}\right) \geqq d_{\Delta}\left(f \circ \varphi_{i}\left(a_{i}\right), f \circ \varphi_{i}\left(b_{i}\right)\right) .
$$

$$
\begin{aligned}
h_{\Delta, K}\left(a_{1}, b_{1}\right) & +\cdots+h_{\Delta, K}\left(a_{n}, b_{n}\right) \\
& \geqq d_{\Delta}\left(f \circ \varphi_{1}\left(a_{1}\right), f \circ \varphi_{1}\left(b_{1}\right)\right)+\cdots+d_{\Delta}\left(f \circ \varphi_{n}\left(a_{n}\right), f \circ \varphi_{n}\left(b_{n}\right)\right) \\
& \geqq d_{\Delta}\left(f\left(p_{0}\right), f\left(p_{1}\right)\right)+\cdots+d_{\Delta}\left(f\left(p_{n-1}\right), f\left(p_{n}\right)\right) \\
& \geqq d_{\Delta}\left(f\left(p_{0}\right), f\left(p_{n}\right)\right)=d_{\Delta}(f(p), f(q)) .
\end{aligned}
$$

Consequently, $d_{D, K}(p, q) \geqq d_{\Delta}(f(p), f(q))$.

THEOREM 2. Let $f: D \rightarrow M$ be a $K$-quasiconformal function on a domain $D$ in a complex Banach space $E$ with values in a hyperbolic Riemann surface. Then $f$ is distance decreasing with respect to the pseudo distances $d_{D, K}$ and $d_{M}$, that is

$$
d_{M I}(f(p), f(q)) \leqq d_{I, K}(p, q), \text { for } p, q \in D .
$$

Proof. The proof is the same as the proof of Theorem 1 except that we replace Theorem $2^{\prime}$ of [4] by Theorem 3 of [4].

The following two theorems are consequences of [4] too.

TheOREM 3 (LiOUVILle's THEOREM). Let $f: E \rightarrow C$ be a bounded $K$ quasiconformal function on a complex Banach space $E$. Then $f$ is a constant.

Theorem 4 (The little Picard theorem). Let $f: E \rightarrow P_{1}(C)-\{3$ points $\}$ be a K-quasiconformal function from a complex Banach space $E$ into the projective line $P_{1}(C)-\{3$ points $\}$. Then $f$ is a constant.

5. A maximum modulus principle. The following theorem implies the usual maximum modulus principle for holomorphic functions.

THEOREM 5. Let $f$ be a $K$-quasiconformal function on a bounded domain $D$ in a complex Banach space E. If $|f(z)|, z \in D$, assumes the maximum value at an interior point in $D$, then $f$ must be a constant.

Proof. We first remark that a nonconstant $K$-quasiconformal function of one complex variable satisfies the maximum modulus principle. Suppose that there is an interior point $z_{0}$ of $D$ at which $|f(z)|$ takes the maximum value $a$. Put

$$
f\left(z_{0}\right)=\alpha, \quad|\alpha|=a \quad \text { and } \quad S=\{z \mid f(z)=\alpha\} .
$$


It is obvious that $S \cap D$ is a nonempty relatively closed set in $D$. But it is also open. Suppose that $\zeta$ is a point in $S \cap D$. For an arbitrary point $z$ in a small neighborhood $U$ of $\zeta$, we have a complex plane passing through $\zeta$ and $z$ which is uniquely determined. On this complex plane, $f$ is $K$-quasiconformal of one complex variable and $|f(z)|$ attains its maximum value at an interior point corresponding to $\zeta$. Therefore $f$ must be a constant on this complex plane so that $z \in S \cap D$. This holds for an arbitrary point $z$ in $U$ which proves that $S \cap D$ is open. Since $D$ is connected, $S \cap D=D$ which implies that the function $f$ is a constant.

\section{REFERENCES}

1. L. V. Ahlfors, Lectures on quasiconformal mappings, Van Nostrand Math. Studies, no. 10, Van Nostrand, Princeton, N.J., 1966. MR 34 \#336.

2. E. Hille and R. S. Phillips, Functional analysis and semi-groups, Amer. Math. Soc. Collog. Publ., vol. 31, Amer. Math. Soc., Providence, R.I., 1957. MR 19, 664.

3. S. Hitotumatu, On quasi-conformal functions of several complex variables, J. Math. Mech. 8 (1959), 77-94. MR 21 \#1398.

4. P. J. Kiernan, Quasiconformal mappings and Schwarz's lemma, Trans. Amer. Math. Soc. 148 (1970), 185-197. MR 41 \#467.

5. S. Kobayashi, Hyperbolic manifolds and holomorphic mappings, Pure and Appl. Math., no. 2, Dekker, New York, 1970. MR 43 \#3503.

6. L. Nachbin, Topology on spaces of holomorphic mappings, Ergebnisse der Mathematik und ihrer Grenzgebiete, Band 47, Springer-Verlag, Berlin and New York, 1969. MR 40 \#7787.

Department of Mathematics, University of Florida, Gainesville, Florida 32601 